\title{
Microsaccades restore the visibility of minute foveal targets
}

Stationary targets can fade perceptually during steady visual fixation, a phenomenon known as Troxler fading. Recent research found that microsaccades—small, involuntary saccades produced during attempted fixation — can restore the visibility of faded targets, both in the visual periphery and in the fovea. Because the targets tested previously extended beyond the foveal area, however, the ability of microsaccades to restore the visibility of foveally-contained targets remains unclear. Here, subjects reported the visibility of low-to-moderate contrast targets contained entirely within the fovea during attempted fixation. The targets did not change physically, but their visibility varied intermittently during fixation, in an illusory fashion (i.e. foveal Troxler fading). Microsaccade rates increased significantly before the targets became visible, and decreased significantly before the targets faded, for a variety of target contrasts. These results support previous research linking microsaccade onsets to the visual restoration of peripheral and foveal targets, and extend the former conclusions to minute targets contained entirely within the fovea. Our findings suggest that the involuntary eye movements produced during attempted fixation do not always prevent fading --in either the fovea or the periphery---and that microsaccades can restore perception, when fading does occur. Therefore, microsaccades are relevant to human perception of foveal stimuli. 
1 Authors: Francisco M. Costela ${ }^{1,2}$, Michael B. McCamy ${ }^{1}$, Stephen Macknik ${ }^{1,3}$, Jorge

2 Otero-Millan ${ }^{1,4}$, Susana Martinez-Conde ${ }^{1^{*}}$

4 Affiliations:

$5 \quad{ }^{1}$ Department of Neurobiology, Barrow Neurological Institute, Phoenix, AZ, USA

$6 \quad{ }^{2}$ Graduate program in Neuroscience, Arizona State University, Tempe, AZ, USA

$7 \quad{ }^{3}$ Department of Neurosurgery, Barrow Neurological Institute, Phoenix, AZ, USA

$8 \quad{ }^{4}$ Department of Signal Theory and Communications, University of Vigo, Spain

"Corresponding author: Susana Martinez-Conde, Department of Neurobiology, Barrow Neurological Institute, 350 W Thomas Rd. Phoenix, AZ 85013, USA. E-mail: smart@neuralcorrelate.com

\section{Introduction}

During attempted fixation, the eyes are not still but continue to produce so called "fixational eye movements”, which include microsaccades, drift and tremor (Martinez-Conde, Macknik, \& Hubel, 2004). In the 1950s, several groups demonstrated that visual perception of stationary objects faded in the absence of eye movements (Ditchburn \& Ginsborg, 1952; Riggs \& Ratliff, 1952; Yarbus, 1957). Thus, fixational eye movements were linked to the prevention of visual fading and restoration of visibility during fixation, although the specific roles of each fixational eye movement type remained controversial (McCamy, et al., 2012). Microsaccades, the largest and fastest fixational eye movement, have been linked to the visual restoration of faded targets (Martinez-Conde, Macknik, Troncoso, \& Dyar, 2006; McCamy, et al., 2012), but their role in peripheral versus central vision has been less clear. eye movement contributor to restoring faded targets, both in the visual periphery and in the fovea (the highest-resolution, rod-free region of the retina, subtending approx. 1.25 degrees of visual angle (Curcio, Sloan, Kalina, \& Hendrickson, 1990)). The target size tested extended beyond the 
31 limits of the fovea, however, and so the ability of microsaccades to restore minute targets

32 contained within the fovea continues to be in question.

33 Here, human subjects reported on the visibility of a target that was centered on the fovea

34 and was smaller than the area of the fovea, during attempted visual fixation. The target did not

35 change physically, but its visibility decreased and increased intermittently during fixation, in an

36 illusory fashion (a perceptual phenomenon known as Troxler fading (Troxler, 1804)).

37 Microsaccade rates increased significantly before the target became visible, and decreased

38 significantly before the target faded, for a variety of target contrasts. These results support

39 previous research linking microsaccade onsets to the visual restoration of peripheral and foveal

40 targets (Martinez-Conde, et al., 2006; McCamy, et al., 2012), and extend the former conclusions

41 to small-size targets that are contained within the fovea.

43 Methods

44 Subjects. Eight subjects (7 males, 1 female) with normal or corrected-to-normal vision

45 participated in the experiments. Six subjects were naive and were paid \$15/session. Experiments

46 were carried out under the guidelines of the Barrow Neurological Institute's Institutional Review

47 Board (protocol number 04BN039). Written informed consent was obtained from each subject.

48

49 Experimental design. Subjects rested their forehead and chin on the EyeLink 1000 head/chin

50 support, $57 \mathrm{~cm}$ away from a linearized video monitor (Barco Reference Calibrator V, $75 \mathrm{~Hz}$

51 refresh rate). The experiment consisted of 4 sessions of $\sim 1$ hour, each including 50 randomly

52 interleaved 30-second trials. The first session was counted as a training session and not included

53 in the analyses. 
While fixating a small red spot $\left(0.03^{\circ}\right.$ diameter $)$ on the center of the screen, subjects

55 continuously reported whether a stimulus was faded/fading (button press) or

56 intensified/intensifying (button release) (Martinez-Conde, et al., 2006; McCamy, et al., 2012,

57 (McCamy et al., 2013)). To start the trial, subjects pressed a key and the stimulus appeared on the

58 screen. Subjects were instructed to release the button as soon as they saw the stimulus. The

59 stimulus was a two-lobe Gabor patch with a peak-to-trough width of $0.5^{\circ}$ (Gaussian standard

60 deviations of $x=0.2^{\circ}$ and $y=0.2^{\circ}$; sine wave period of $1^{\circ}$; sine wave phase of 0 ). The Gabor was

61 presented at the center of the screen and contained within the fovea, with contrast levels of 5\%,

$6210 \%, 20 \%$, and $40 \%$ from peak-to-trough and the same average luminance (50\%) as the

63 background. The orientation of the Gabor varied randomly between $0^{\circ}$ and $360^{\circ}$ in each trial, to

64 control for orientation adaptation effects (Martinez-Conde, et al., 2006; McCamy, et al., 2012).

65 After 30 seconds, the stimuli disappeared and the trial ended.

67 Eye movement analyses. Eye position was acquired noninvasively with a fast video-based eye

68 tracker at $500 \mathrm{~Hz}$ (EyeLink 1000, SR Research). We recorded eye movements simultaneously in

69 both eyes (instrument noise $0.01^{\circ} \mathrm{RMS}$ ). We identified and removed blink periods as portions of

70 the raw data where pupil information was missing. We also removed portions of data where very

71 fast decreases and increases in pupil area occurred (> 50 units/sample, such periods are probably

72 semi-blinks where the pupil is never fully occluded) (Troncoso, Macknik, \& Martinez-Conde,

73 2008). We added 200 ms before and after each blink/semi-blink to eliminate the initial and final

74 parts where the pupil was still partially occluded (Troncoso, Macknik, \& Martinez-Conde, 2008).

75 Saccades were identified with a modified version of the algorithm developed by Engbert \&

76 Kliegl (Engbert, 2006; Engbert \& Kliegl, 2003; Engbert \& Mergenthaler, 2006; Laubrock, 
77 Engbert, \& Kliegl, 2005; Rolfs, Laubrock, \& Kliegl, 2006) with $\lambda=4$ (used to obtain the

78

79

80

81

82

velocity threshold) and a minimum saccadic duration of 6 ms. To reduce the amount of potential noise, we considered only binocular saccades, that is, saccades with a minimum overlap of one data sample in both eyes (Engbert, 2006; Engbert \& Mergenthaler, 2006; Laubrock, et al., 2005; Rolfs, et al., 2006). Additionally, we imposed a minimum intersaccadic interval of $20 \mathrm{~ms}$ so that potential overshoot corrections might not be categorized as new saccades (Moller, Laursen, Tygesen, \& Sjolie, 2002). Microsaccades were defined as saccades with magnitude $<1^{\circ}$ in both eyes (Martinez-Conde, Macknik, Troncoso, \& Hubel, 2009; Martinez-Conde, Otero-Millan, \& Macknik, 2013). To calculate microsaccade properties such as magnitude and peak velocity we averaged the values for the right and left eyes. Figure 1A-B shows the magnitude distribution (Figure 1A) and peak velocity-magnitude relationship (Figure 1B) for both microsaccades $\left(<1^{\circ}\right.$ ) and saccades $\left(\geq 1^{\circ}\right)$. All subsequent analyses (Figures 3-5) concern microsaccades only, that is, saccades with magnitude $<1^{\circ}$.

\section{<< INSERT FIGURE 1 HERE $>>$}

Figure 1. Descriptive statistics for microsaccades $\left(<1^{\circ}\right)$ and saccades $\left(\geq 1^{\circ}\right)$. A) Microsaccadic and saccadic magnitude distribution for all subjects combined $(n=8)$. B) Microsaccadic and saccadic peak velocity-magnitude relationship for all subjects combined. Each orange dot represents a microsaccade or a saccade with peak velocity indicated on the $y$-axis and magnitude indicated on the $x$-axis. The inset legend shows the microsaccade descriptive statistics. Error bars and shadows indicate the SEM across subjects.

Microsaccade correlations with reported transitions. Let $X_{M}, X_{S}, X_{B}$, and $X_{R}$ be the stochastic processes representing the onsets of microsaccade, and intensification report $(R)$. For example, if 
$S_{1}, S_{2}, \cdots, s_{k}$

102

are the start times of all the microsaccades for a given subject, then $X_{M}$ for that

103 subject will be given by

$$
X_{M}(t)=1 \quad t=s_{i} \quad 1 \leq i \leq k, \quad X_{M}(t)=0
$$

if for some and

otherwise;

104 similarly for intensification reports.

105 We obtained correlations of microsaccades with reports of intensification for each

$$
\xi_{M R}(t)=\sum_{n=-\infty}^{n=\infty} X_{M}(n+t) X_{R}(n)
$$

106 subject, using

and then converting it to a rate (similarly for

107 transitions to fading) (McCamy, et al., 2012). For each subject, correlations were smoothed using

108 a Savitzky-Golay filter of order 1 and a window size of 151 ms (Martinez-Conde, et al., 2006).

109 Average correlations are the average of the smoothed correlations (Figures 3-4).

110

111 ROC analysis. We used a sliding receiver operating characteristic (ROC) analysis (Britten,

112 Shadlen, Newsome, \& Movshon, 1992; Feierstein, Quirk, Uchida, Sosulski, \& Mainen, 2006;

113 Green \& Swets, 1966; Hernandez, Zainos, \& Romo, 2002; Romo, Hernandez, \& Zainos, 2004;

114 Romo, Hernandez, Zainos, Lemus, \& Brody, 2002; Troncoso, Macknik, Otero-Millan, \&

115 Martinez-Conde, 2008) to quantify how well microsaccade rate may predict the type of

116 perceptual transition (towards intensification versus fading) reported by the subjects, for the

117 different foveal target contrasts. The area under an ROC curve provides a measure of the

118 discriminability of two signals and is directly related to the overlap of the two distributions of

119 responses that are compared (Feierstein, et al., 2006). In our case, the area under the ROC curve

120 can be interpreted as the probability with which an ideal observer, given the microsaccade rate 
121 during a window of time around a particular transition, can correctly determine the type of 122 transition (towards fading or intensification). An ROC area of 0.5 corresponds to completely 123 overlapping distributions (the ideal observer cannot discriminate between the two types of 124 transitions); an area of 1 corresponds to transitions that can be perfectly discriminated based on 125 microsaccade rate. This analysis makes no assumptions about the underlying distributions 126 (Feierstein, et al., 2006). For a given point in time, we compared the microsaccade rate 127 distributions for transitions to intensification (true-positive rate) and transitions to fading 128 (false-positive rate) for each subject. To obtain the ROC curve at that time, we plotted the 129 probability of true positives as a function of the probability of false positives for all possible 130 criterion response levels. We performed a sliding ROC analysis (kernel width 500 ms, slid in 131 2-ms increments) to calculate each subject's area under the ROC curve at each time point around 132 the transition. To determine the time point at which the ideal observer became better than chance, 133 we calculated significance using a permutation procedure (Feierstein, et al., 2006; Hernandez, et 134 al., 2002; Romo, et al., 2004; Romo, et al., 2002; Siegel \& Castellan, 1988; Troncoso, Macknik,

135 Otero-Millan, et al., 2008) with $n=1,000$ shuffles for each subject and a criterion $p$-value $<0.01$.

136 Statistical methods. To analyze the effect of target contrast on time faded per trial and rate of

137 fading onsets (Figure 2A-B), we conducted separate single-factor repeated measures ANOVAs 138 with the different contrast levels tested as the within-subjects factor. All post-hoc comparisons 139 were done using Tukey's HSD method. To assess whether microsaccade rates before transitions 140 to intensification were significantly higher than those before transitions to fading, we performed 141 one-tailed paired $t$-tests in each bin (bin size $=20 \mathrm{~ms}$ ), using Bonferroni correction to account for 142 multiple comparisons (Figures 3-4). Significance levels were set to $\alpha=0.01$ throughout.

\section{Results}


144 Subjects fixated a small spot on the center of a computer screen and continuously reported, via

145 button press/release, whether an unchanging visual target (a 2-lobe Gabor patch with 5\%, 10\%,

$14620 \%$, or $40 \%$ contrast), presented within the fovea, was faded (or in the process of fading) versus

147 intensified (or intensifying) (Martinez-Conde, et al., 2006; McCamy, et al., 2012). Fading

148 prevalence decreased as the target's contrast increased (Figure 2A), and the 10\% contrast target

149 generated the largest number of perceptual transitions, as indicated by subjects' reports (Figure

150 2B). As expected, lower-contrast targets were faded for longer time periods than higher contrast

151 targets; thus the $5 \%$ contrast target resulted in the longest fading periods and the $40 \%$ contrast

152 target in the longest intensification periods, whereas the $10 \%$ contrast target produced fading and

153 intensification periods of comparable length (Figure 2C-F).

154

155

$<<$ INSERT FIGURE 2 HERE $>>$

156

157 Figure 2. Perceptual reports. A) Average time faded per trial for each target contrast. The time 158 faded per trial decreased linearly with target contrast $(F(3,21)=88.48, p<0.001$; linear trend $159 F(1,7)=112.36, p<0.0001)$. B) Fading onset rate for each target contrast. The effect of contrast 160 was significant $(F(3,21)=8.58, p=0.0065)$. A Tukey HSD posthoc comparison showed a 161 significant difference only between the $10 \%$ and $40 \%$ contrast target $(p<0.01)$. C-F)

162 Distribution of the durations of intensification and fading periods for each target contrast. Error 163 bars and red and blue shadows indicate the SEM across subjects $(n=8)$.

164

165

166

167

168

Microsaccade rates increased before transitions to intensification and decreased before transitions to fading, with the intermediate-contrast targets (10\% and $20 \%)$ showing the strongest correlations between microsaccade rate increases and intensification reports (Figure 3). 
170 Figure 3. Microsaccade correlations with reported transitions. A-D) Percent increase in microsaccade rate over baseline (i.e. relative to the average microsaccade rate (dashed horizontal line) for a given target contrast) around reported transitions toward intensification versus fading, for each target contrast. The solid vertical line indicates the reported transitions $(t=0)$. Target contrast is indicated at the top of each panel. The gray lines at the top indicate the bins where microsaccade rates before transitions to intensification were significantly higher than microsaccade rates before transitions to fading (see Methods for details). Red and blue shadows indicate the SEM across subjects $(n=8)$.

Microsaccade magnitude. We analyzed the effects of microsaccade size on perceptual

transitions to intensification and fading. To do this, we separated all microsaccades in 4 different

181

categories according to size (0-15 arcmin, 15-30 arcmin, 30-45 arcmin, and 45-60 arcmin) and

correlated them to the perceptual intensification and fading reports for each target contrast

(Figure 4). The smallest microsaccades failed to restore target visibility, especially for those

targets with the lowest levels of contrast (not shown). This result is consistent with the previous

increased ability to bring the neuronal receptive fields to uncorrelated stimulus regions

(McCamy, et al., 2012). As microsaccades grew in size, their correlation with perceptual

transitions became stronger, also consistent with previous research (McCamy, et al., 2012)).

\section{$<<$ INSERT FIGURE 4 HERE $>>$}

Figure 4. Correlations between microsaccades of different sizes and reported transitions. Microsaccade sizes (0-15 arcmin; 15-30 arcmin; 30-45 arcmin; 45-60 arcmin) are indicated at the top of each panel and we have collapsed across target contrasts. All other details as in Figure 196

197 Receiver operating characteristic (ROC) analysis. To further quantify our conclusions, we 198 conducted a sliding ROC analysis to calculate the ability of an ideal observer to predict the type 
of perceptual transition (towards intensification or fading) based on microsaccade rates. Figure 5

200

201

202

203

204

205

206

207

208

209

210

211

212

213

214

215

216

217

218

219

220

221

222

223

224

225

shows that the ideal observer becomes significantly better than chance (determined by

permutation analysis; see Methods for details) $\approx 800$ ms before the reported transitions, for targets of $10 \%, 20 \%$, and $40 \%$ contrast.

\section{$<<$ INSERT FIGURE 5 HERE $>>$}

Figure 5. ROC analysis. A-C) The ideal observer can predict the type of illusory transitions (intensification vs. fading) based on microsaccade rate. The green line is the area under the ROC curve at any given time. The solid horizontal gray line indicates the significance level (i.e. the level at which the ideal observer performs above chance (horizontal dashed line; see Methods for details). Target contrast is indicated at the top of each panel. Significance is reached whenever the green line is above the grey line. The ideal observer's prediction (green line) becomes significantly better than chance $\approx 800$ ms before the reported transitions, for target contrasts of $10 \%, 20 \%$ and $40 \%$. The shaded green area indicates the SEM across subjects $(n=8)$.

\section{Discussion}

In 1804, Troxler described the perceptual fading of stationary objects during fixation, a

perceptual phenomenon that came to be known as Troxler fading (Troxler, 1804). Despite

Troxler's report that not only peripheral, but also centrally fixated targets were susceptible to fading, Troxler fading became equated with peripheral fading in subsequent decades (see (Wade \& Tatler, 2005) for a historical review). Yet, foveal fading has been reported by numerous researchers in a number of experimental conditions (Darwin, 1795; Krauskopf, 1963; McCamy, et al., 2012; Pessoa \& De Weerd, 2003; Simons, et al., 2006; Troxler, 1804). A recent study found that microsaccades counteracted the perceptual (i.e. Troxler) fading of peripherally and foveally presented Gabor patches with peak-to-trough widths of $2.5^{\circ}$ (McCamy, et al., 2012). Because the edges of such centrally presented targets extended beyond the foveal limits, here we asked 
226

227

228

229

230

231

232

233

234

235

236

237

238

239

240

241

242

243

244

245

246

247

248

whether smaller size targets, constrained to the area of the fovea, might similarly fade from perception, and if so, whether microsaccades could restore their visibility as well.

Subjects reported the visibility of centrally presented 2-lobe Gabor patches with

peak-to-trough widths of $0.5^{\circ}$ (that is, 5 times smaller than in (McCamy, et al., 2012)), of varying

contrasts (5\%,10\%, 20\%, 40\%). As with previous fading experiments (Martinez-Conde, et al.,

2006; McCamy, et al., 2012; Spillmann \& Kurtenbach, 1992; Troncoso, Macknik, \&

Martinez-Conde, 2008), subjects reported that the perceptual state of the (foveally contained)

targets appeared to oscillate between the faded/fading state and the visible/intensifying state.

Thus, minute targets constrained to the fovea are subject to perceptual fading during fixation, in

much the same manner as larger, foveally centered targets, and peripherally presented targets are

(Martinez-Conde, et al., 2006; McCamy, et al., 2012). Microsaccade rates increased before

transitions to visibility and decreased before transitions to invisibility, also in agreement with

previous reports (Martinez-Conde, et al., 2006; McCamy, et al., 2012; Troncoso, Macknik, \&

Martinez-Conde, 2008). These findings indicate that microsaccades have comparable effects in

visual target restoration across the retina, for a variety of target sizes and contrasts.

\section{Target contrast and foveal fading}

As one might have expected, lower-contrast targets faded more often and for longer amounts of time than higher-contrast targets (Figure 2). Perhaps more surprisingly, targets of moderate contrast levels (i.e. $20 \%$ and $40 \%$ ) also faded, albeit less often, and microsaccades restored them perceptually at such times (Figures 2-3, 5). The correlation between microsaccade production and visual restoration of faded targets was most obvious for the intermediate contrast targets (i.e. 10\% and 20\%), although still present for lower and higher contrasts (i.e. 5\% and 40\%) (Figures 3 and 5). Why did microsaccades restore the visibility of low-contrast (i.e. 5\%) targets less 
249 effectively than that of intermediate-contrast targets? It seems likely that, when target visibility is 250 highly degraded (for instance, due to minimum contrast levels), shifting of the retinal image due

251 to microsaccades or other eye movements may not produce enough photoreceptor stimulation to 252 generate a perceptual experience in a reliable way. Conversely, when intermediate or moderate 253 contrast targets fade from perception, their visibility may be degraded just enough that 254 microsaccades are able to restore them with high efficiency.

255 The current results are consistent with previous evidence that stimuli do fade at all retinal 256 eccentricities (Wade \& Tatler, 2005), despite the concerted actions of the three types of fixational 257 eye movements (i.e. microsaccades, drift and tremor), and that microsaccades can successfully 258 bring back such faded stimuli (McCamy, et al., 2012).

259

260

Are microsaccades relevant to foveal visibility?

261 The present results show that microsaccades can restore the visibility of small, foveally 262 contained targets of moderate-to-low (i.e. ranging from approximately $40 \%$ to $10 \%$ ) contrasts. Very low contrast targets (i.e. 5\% contrast) were visible for a small amount of the time only, and microsaccades did not significantly restore their visibility (although there was a trend between microsaccade production and target intensification (Figures 3-5), especially for the larger microsaccade sizes (not shown)). High-contrast targets (i.e. higher than $40 \%$ contrast) were not tested in this study, but the current data suggest that they would have faded too briefly and rarely

268 for microsaccades to restore their visibility in a substantial manner.

269

In light of these combined results, what is the value of microsaccades to foveal

270 perception? We posit that—despite assumptions that only high-contrast stimuli are pertinent to

271 foveal vision — most visible stimuli are relevant to perception by definition, regardless of their 
272 contrast. That is, fading of any stimulus (i.e. irrespective of contrast) is a visual degradation that

273 microsaccades often supersede. Indeed, there are many small-sized, low-contrast objects that one

274 might want to see optimally with central vision. A diamond earring on a white carpet, or small

275 features in medical and research images, are two of many examples. Thus, the fovea has the

276 capacity to inspect stimuli of all contrasts and spatial frequencies, and microsaccades can restore

277 the visibility of a range of such foveal stimuli, after fading sets in. Therefore, microsaccades are 278 relevant to human perception of foveal stimuli.

279 Future research should investigate the ability of microsaccades to restore faded targets of 280 diverse spatial frequency content, at varied retinal eccentricities.

281

282 On the visibility of fixation targets

283 One frequent argument against the value of microsaccades to foveal vision is that fixation 284 targets, which are presented centrally, never fade, even in the absence of microsaccades. We note 285 that the sizes, colors and shapes of fixation targets used in vision studies vary widely (McCamy, 286 Najafian Jazi, Otero-Millan, Macknik, \& Martinez-Conde, 2013; Thaler, Schutz, Goodale, \& 287 Gegenfurtner, 2013), but their contrasts (and high spatial frequency content) are almost 288 universally maximized. The present results suggest that fixation targets remain perpetually visible by virtue of their high contrast (and possibly high spatial frequency), rather than their 290 small size and/or foveal presentation. Further, our results show that it is possible for one foveal

291 stimulus to fade from perception (i.e. the low-to-moderate contrast Gabor patches presented 292 here) and for another foveal stimulus to remain visible (i.e. the fixation target), at the same time. 293

\section{Complete fading versus partial loss of visibility during fixation}


295 Troxler fading, the perceptual experience at the heart of the present study, is a gradual, rather

296 than an instantaneous process. Often, an object becomes less and less visible until it eventually

297 disappears (and then reappears, typically when microsaccades bring it back, as shown here).

298 Other times, an object's visibility decreases at first, and then it is restored (again, usually in

299 connection with microsaccade production) before complete fading has occurred. The current

300 research set out to quantify the precise timing of the interactions between microsaccades and

301 perceptual experience (similarly to Martinez-Conde et al., 2006; McCamy et al., 2012). Thus,

302 experimental subjects indicated when the target was faded/fading versus intensified/intensifying,

303 rather reported merely when the target was completely faded versus fully visible. Had we

304 considered only "total fading" and disregarded "partial fading" events, we would have achieved

305 an incomplete picture of the role of microsaccades in visual restoration, rather than the full,

306 dynamic picture of the interactions between microsaccade production and the ongoing perceptual

307 experience that characterizes natural vision. Future research may investigate how microsaccades

308 and other fixational eye movements impact the perception of gradations in fading/visibility (i.e.

309 by obtaining a continuous measure of the subject's perceptual experience, as in Simons et al.,

310 2006), rather than focus on the perceptual transitions to increased or decreased visibility (i.e. as

311 in the present paradigm).

312

313 Fading prevention versus visibility restoration

314 Fixational eye movements are thought to overcome loss of vision by thwarting the neural

315 adaptation (and thus the visual fading) ensuing from stable stimulation of the retinal receptors

316 (Martinez-Conde et al., 2004). A fruitful discussion of the perceptual effects of microsaccades-

317 in central vision and at other retinal eccentricities—must separately address their impact on 
318 counteracting (that is, reversing) fading versus preventing the fading from occurring in the first

319 place (Martinez-Conde, et al., 2013). Here we set out to address the ability of microsaccades to 320 counteract (i.e. reverse) fading; that is, to restore the visibility of already faded objects. Future

321 research should establish the ability of the different types of fixational eye movements to prevent 322 rather than counteract fading (i.e. to prevent vision loss versus restore faded vision), as well as 323 the physiological mechanisms by which fixational eye movements prevent and counteract neural 324 adaptation.

325 Previous studies found that drift does not contribute strongly to reversing fading

326 (Martinez-Conde, et al., 2006; McCamy, et al., 2012). Whereas microsaccades counteract fading 327 once it has occurred, it is possible that both microsaccades and drift work together to prevent 328 fading before it happens. Future research should also investigate this hypothesis.

329 In sum, fixational eye movements serve to prevent fading in the fovea and elsewhere, but 330 not perfectly. Microsaccades have the ability to bring stimuli back to perception, when peripheral 331 and foveal fading do occur. 


\section{Acknowledgements:}

333 This study was also supported by the Barrow Neurological Foundation (to SLM and SMC) and 334 the National Science Foundation (Awards 0852636 and 1153786 to SMC and Award 0726113 to 335 SLM). We thank Behrooz Kousari for technical assistance.

336

337

338

339

340

341

342

343

344 345 346

347

348

349

350

351

352

353

354

355

356

357

\section{References}

Britten, K. H., Shadlen, M. N., Newsome, W. T., \& Movshon, J. A. (1992). The analysis of visual motion: a comparison of neuronal and psychophysical performance. J Neurosci, 12(12), 4745-4765.

Curcio, C. A., Sloan, K. R., Kalina, R. E., \& Hendrickson, A. E. (1990). Human photoreceptor topography. J Comp Neurol, 292(4), 497-523.

Darwin, E. (1795). Zoonomia; or the laws of organic life, in three parts (4th American ed. ed. Vol. 2). Philadelphia: Edward Earle.

Ditchburn, R. W., \& Ginsborg, B. L. (1952). Vision with a stabilized retinal image. Nature, 170, 36-37.

Engbert, R. (2006). Microsaccades: A microcosm for research on oculomotor control, attention, and visual perception. Prog Brain Res, 154, 177-192.

Engbert, R., \& Kliegl, R. (2003). Microsaccades uncover the orientation of covert attention. Vision Res, 43(9), 1035-1045.

Engbert, R., \& Mergenthaler, K. (2006). Microsaccades are triggered by low retinal image slip. Proc Natl Acad Sci U S A, 103(18), 7192-7197.

Feierstein, C. E., Quirk, M. C., Uchida, N., Sosulski, D. L., \& Mainen, Z. F. (2006). Representation of spatial goals in rat orbitofrontal cortex. Neuron, 51(4), 495-507.

Green, D. M., \& Swets, J. A. (1966). Signal detection theory and psychophysics. New York: Wiley.

Hernandez, A., Zainos, A., \& Romo, R. (2002). Temporal evolution of a decision-making process in medial premotor cortex. Neuron, 33(6), 959-972. 
358

359

360

361

362

363

364

365

366

367

368

369

370

371

372

373

374

375

376

377

378

379

380

381

382

Krauskopf, J. (1963). Effect of retinal image stabilization on the appearance of heterochromatic targets. $J$ Opt Soc Am, 53, 741-744.

Laubrock, J., Engbert, R., \& Kliegl, R. (2005). Microsaccade dynamics during covert attention. Vision Res, 45(6), 721-730.

Martinez-Conde, S., Macknik, S. L., \& Hubel, D. H. (2004). The role of fixational eye movements in visual perception. Nature Reviews Neuroscience, 5, 229-240.

Martinez-Conde, S., Macknik, S. L., Troncoso, X., \& Dyar, T. A. (2006). Microsaccades counteract visual fading during fixation. Neuron, 49, 297-305.

Martinez-Conde, S., Macknik, S. L., Troncoso, X. G., \& Hubel, D. H. (2009). Microsaccades: a neurophysiological analysis. Trends in Neurosciences, 709.

Martinez-Conde, S., Otero-Millan, J., \& Macknik, S. L. (2013). The impact of microsaccades on vision: towards a unified theory of saccadic function. Nature Reviews Neuroscience, 14, 83-96.

McCamy, M. B., Najafian Jazi, A., Otero-Millan, J., Macknik, S. L., \& Martinez-Conde, S. (2013). The effects of fixation target size and luminance on microsaccades and square-wave jerks. PeerJ, in press.

McCamy, M. B., Collins, N., Otero-Millan, J., Al-Kalbani, M., Macknik, S. L., Coakley, D., ... Martinez-Conde, S. (2013). Simultaneous recordings of ocular microtremor and microsaccades with a piezoelectric sensor and a video-oculography system. PeerJ, 1, e14. doi:10.7717/peerj.14

McCamy, M. B., Otero-Millan, J., Macknik, S. L., Yang, Y., Troncoso, X. G., Baer, S. M., et al. (2012). Microsaccadic efficacy and contribution to foveal and peripheral vision. J Neurosci, 32(27), 9194-9204.

Moller, F., Laursen, M. L., Tygesen, J., \& Sjolie, A. K. (2002). Binocular quantification and characterization of microsaccades. Graefes Arch Clin Exp Ophthalmol, 240(9), 765-770.

Pessoa, L., \& De Weerd, P. (Eds.). (2003). Filling-in: from perceptual completion to cortical reorganization. New York: Oxford University Press. 
383

384

385

386

387

388

389

390

391

392

393

394

395

396

397

398

399

400

401

402

403

404

405

406

407

408

Riggs, L. A., \& Ratliff, F. (1952). The effects of counteracting the normal movements of the eye. J. Opt. Soc. Am., 42, 872-873.

Rolfs, M., Laubrock, J., \& Kliegl, R. (2006). Shortening and prolongation of saccade latencies following microsaccades. Exp Brain Res, 169(3), 369-376.

Romo, R., Hernandez, A., \& Zainos, A. (2004). Neuronal correlates of a perceptual decision in ventral premotor cortex. Neuron, 41(1), 165-173.

Romo, R., Hernandez, A., Zainos, A., Lemus, L., \& Brody, C. D. (2002). Neuronal correlates of decision-making in secondary somatosensory cortex. Nat Neurosci, 5(11), 1217-1225.

Siegel, S., \& Castellan, N. J. (1988). Nonparametric statistics for behavioral sciences. New York: McGraw-Hill.

Simons, D., Lleras, A., Martinez-Conde, S., Slichter, D., Caddigan, E., \& Nevarez, G. (2006). Induced visual fading of complex images Journal of Vision, 6, 1093-1101.

Spillmann, L., \& Kurtenbach, A. (1992). Dynamic noise backgrounds facilitate target fading. Vision Research, 32(10), 1941-1946.

Thaler, L., Schutz, A. C., Goodale, M. A., \& Gegenfurtner, K. R. (2013). What is the best fixation target? The effect of target shape on stability of fixational eye movements. Vision Res, 76, 31-42.

Troncoso, X., Macknik, S. L., \& Martinez-Conde, S. (2008). Microsaccades counteract perceptual filling-in. Journal of Vision, 8 (14):15, 1-9.

Troncoso, X., Macknik, S. L., Otero-Millan, J., \& Martinez-Conde, S. (2008). Microsaccades drive illusory motion in the Enigma illusion. Proceedings of the National Academy of Sciences U.S.A., 105, 16033-16038.

Troxler, D. (1804). Ueber das Verschwinden gegebener Gegenstände innerhalb unseres Gesichtskreises. In H. K. u. S. J.A. (Ed.), Ophthalmologische Bibliothek (Vol. II.2, pp. 1-53). Jena: Springer.

Wade, N., \& Tatler, B. W. (2005). The moving tablet of the eye: the origins of modern eye movement research. New York: Oxford UP.

Yarbus, A. L. (1957). The perception of an image fixed with respect to the retina. Biophysics, 2, 683-690. 


\section{Figure 1}

Descriptive statistics for microsaccades $\left(<1^{\circ}\right)$ and saccades $\left(\geq 1^{\circ}\right)$.

A) Microsaccadic and saccadic magnitude distribution for all subjects combined $(n=8)$. B)

Microsaccadic and saccadic peak velocity-magnitude relationship for all subjects combined. Each

orange dot represents a microsaccade or a saccade with peak velocity indicated on the $y$-axis and magnitude indicated on the $x$-axis. The inset legend shows the microsaccade descriptive statistics.

Error bars and shadows indicate the SEM across subjects.
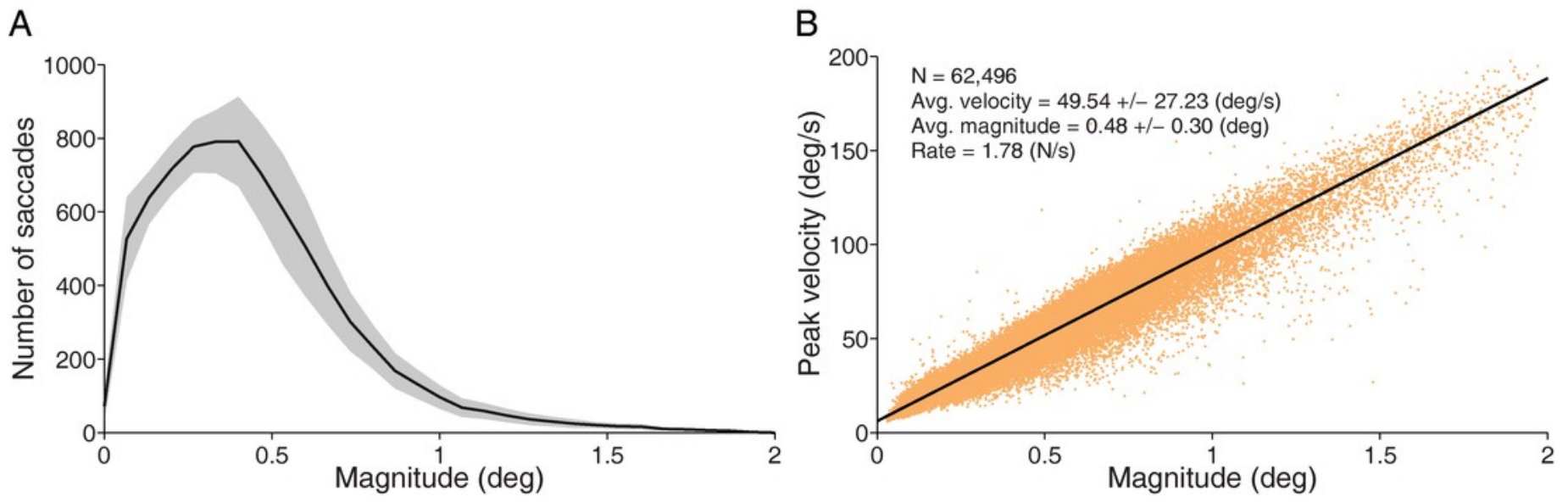


\section{Figure 2}

Perceptual reports.

A) Average time faded per trial for each target contrast. The time faded per trial decreased linearly with target contrast $(F(3,21)=88.48, p<0.001$; linear trend $F(1,7)=112.36, p<0.0001)$. B) Fading onset rate for each target contrast. The effect of contrast was significant $(F(3,21)=8.58, p=0.0065)$. A Tukey HSD posthoc comparison showed a significant difference only between the $10 \%$ and $40 \%$ contrast target $(p<0.01)$. C-F) Distribution of the durations of intensification and fading periods for each target contrast. Error bars and red and blue shadows indicate the SEM across subjects $(n=8)$. 
A

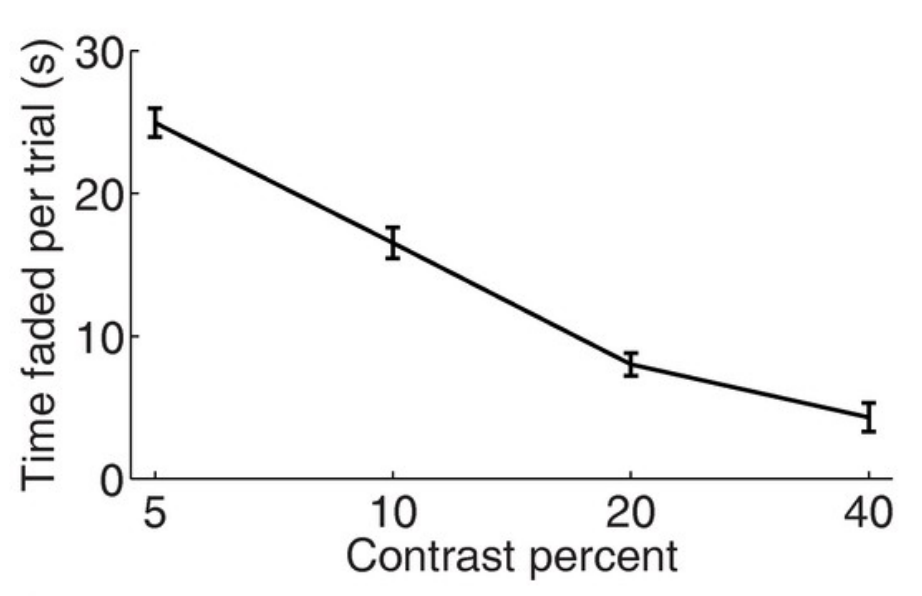

C

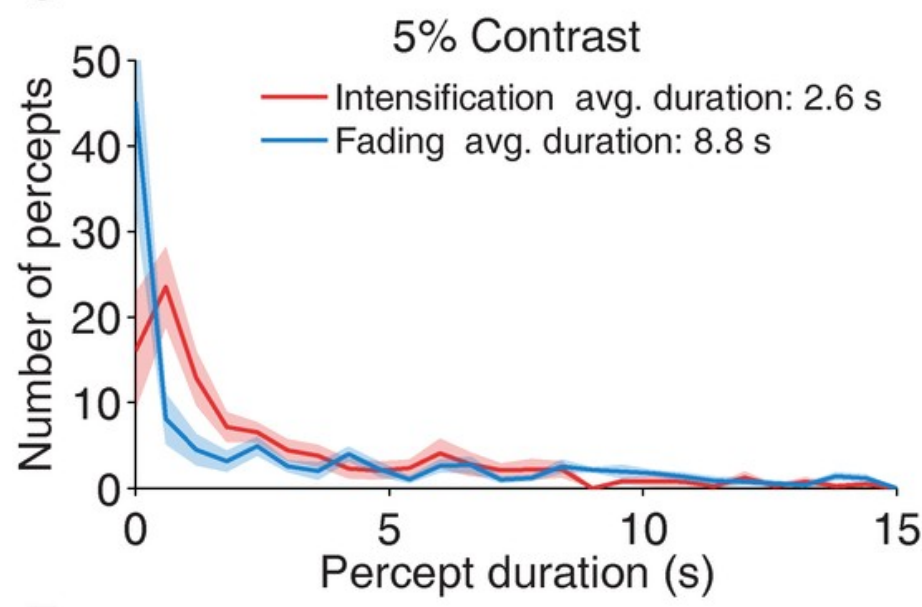

E

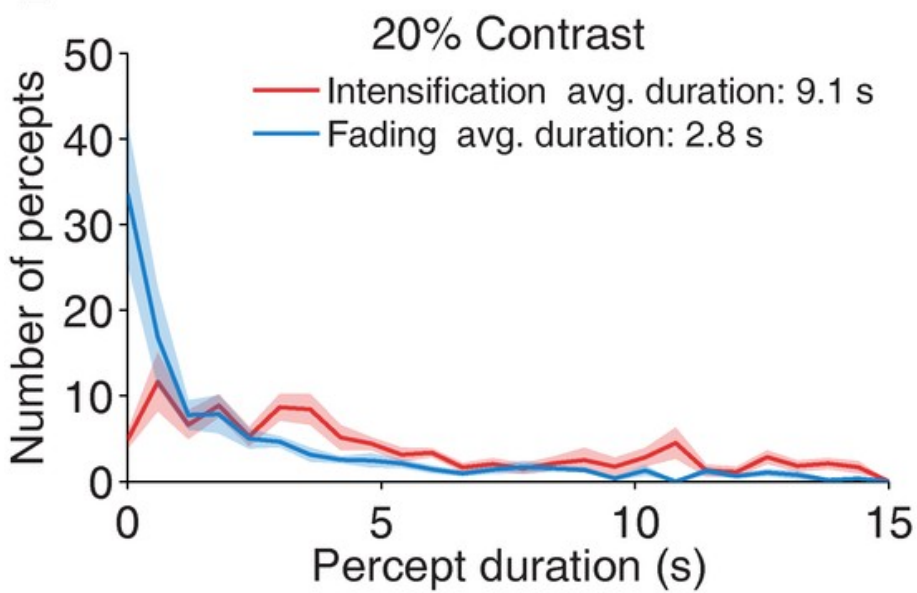

B

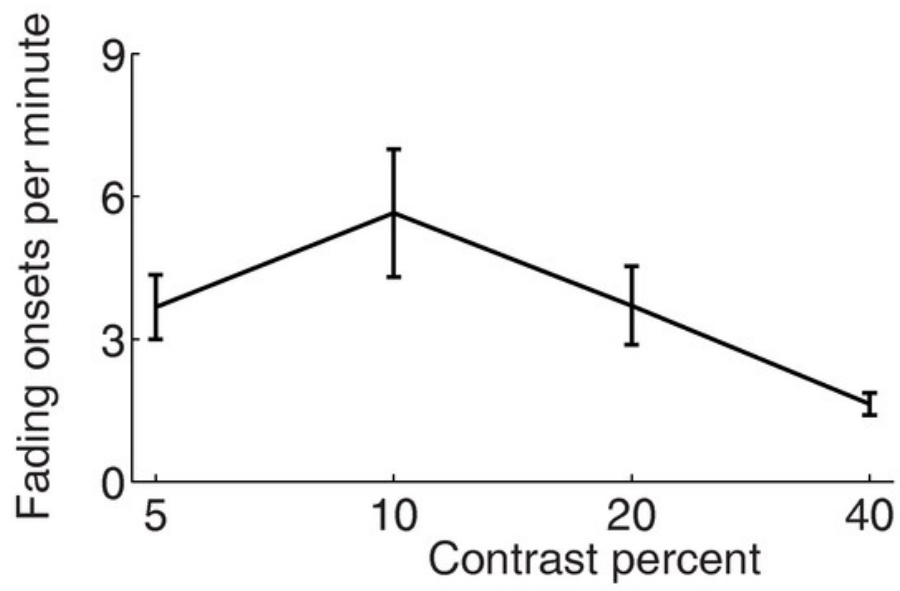

D

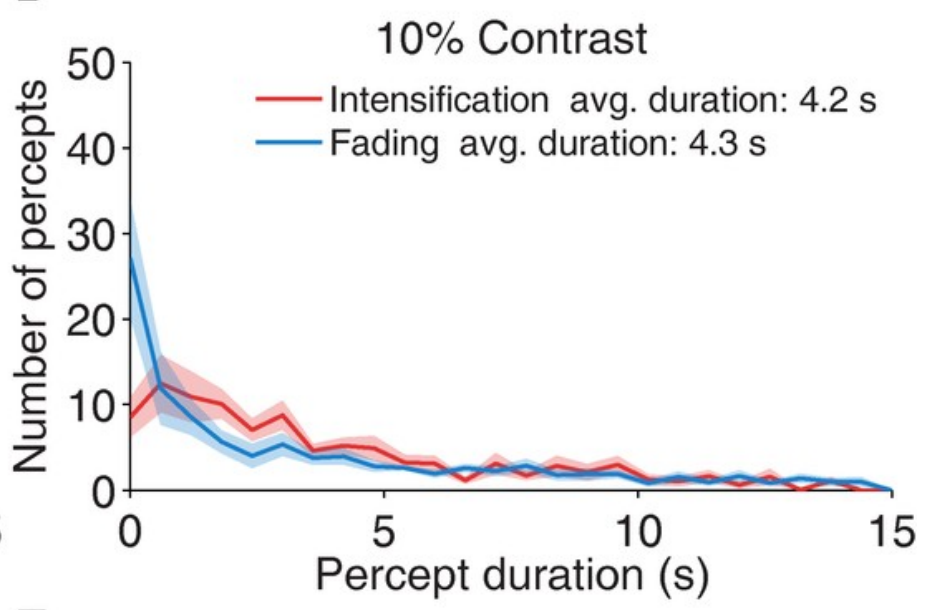

F

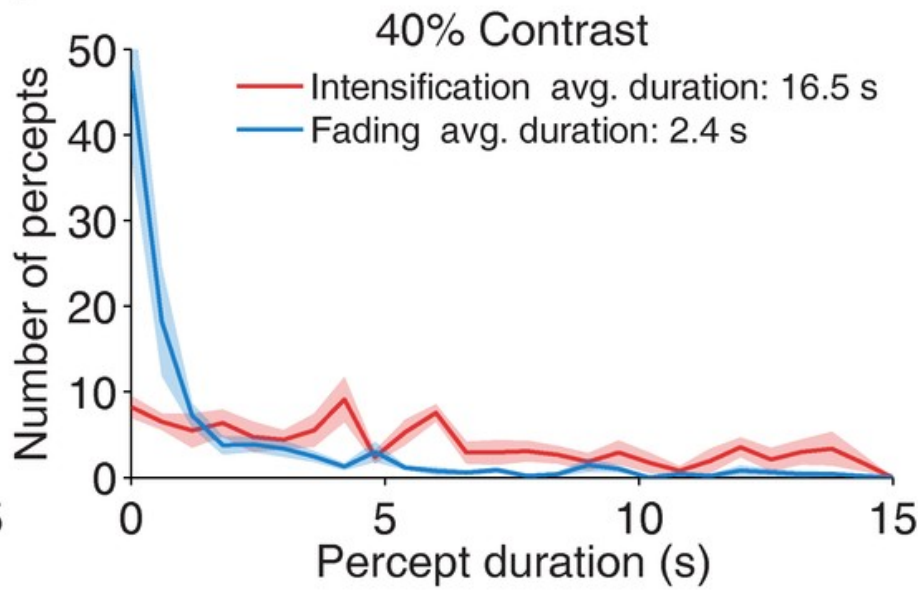




\section{Figure 3}

Microsaccade correlations with reported transitions.

A-D) Percent increase in microsaccade rate over baseline (i.e. relative to the average microsaccade rate (dashed horizontal line) for a given target contrast) around reported transitions toward intensification versus fading, for each target contrast. The solid vertical line indicates the reported transitions $(t=0)$. Target contrast is indicated at the top of each panel. The gray lines at the top indicate the bins where microsaccade rates before transitions to intensification were significantly higher than microsaccade rates before transitions to fading (see Methods for details). Red and blue shadows indicate the SEM across subjects $(n=8)$.

A



C

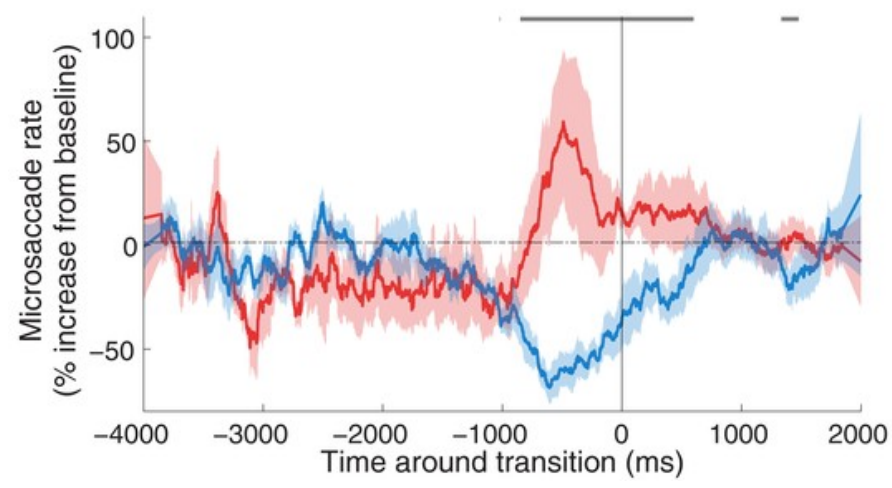

B

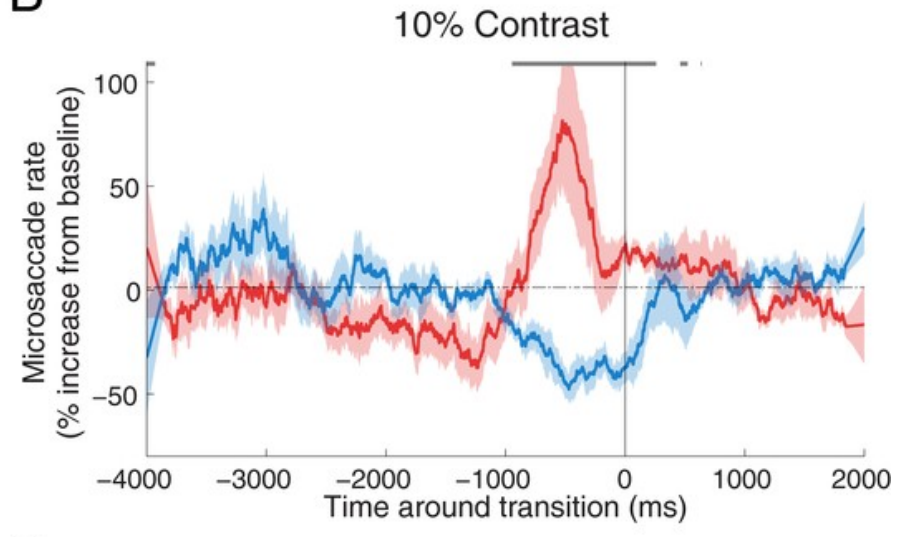

D $\quad 40 \%$ Contrast

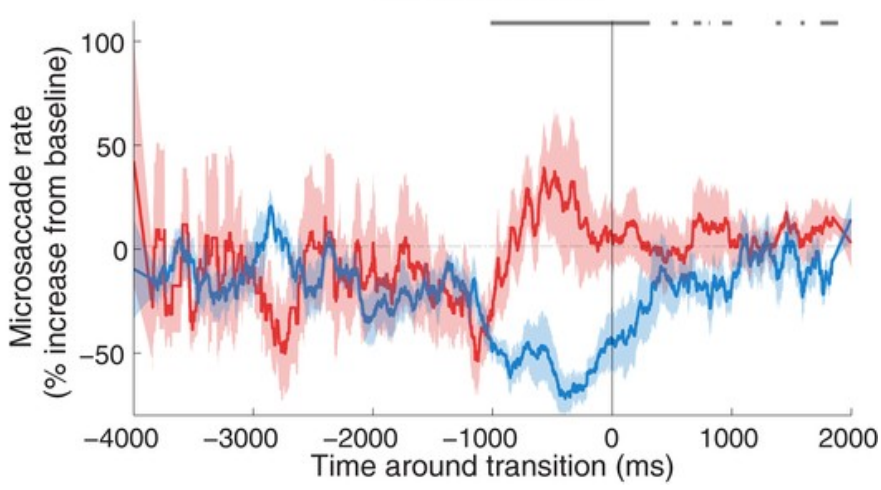




\section{Figure 4}

Correlations between microsaccades of different sizes and reported transitions.

Microsaccade sizes (0-15 arcmin; 15-30 arcmin; 30-45 arcmin; 45-60 arcmin) are indicated at the top of each panel and we have collapsed across target contrasts. All other details as in Figure 3.
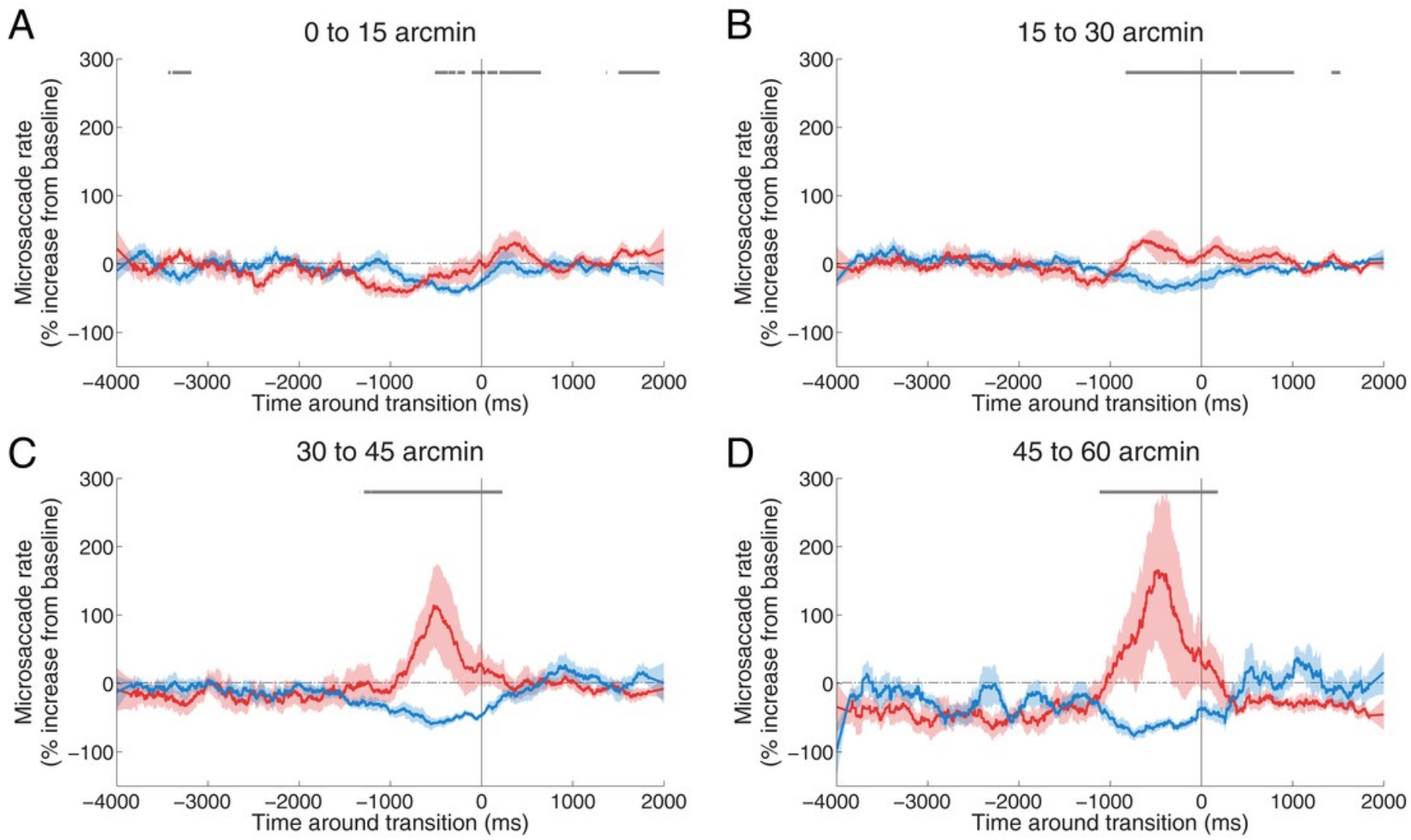


\section{Figure 5}

ROC analysis.

A-C) The ideal observer can predict the type of illusory transitions (intensification vs. fading) based on microsaccade rate. The green line is the area under the ROC curve at any given time. The solid horizontal gray line indicates the significance level (i.e. the level at which the ideal observer performs above chance (horizontal dashed line; see Methods for details). Target contrast is indicated at the top of each panel. Significance is reached whenever the green line is above the grey line. The ideal observer's prediction (green line) becomes significantly better than chance $\approx 800$ ms before the reported transitions, for target contrasts of $10 \%, 20 \%$ and $40 \%$. The shaded green area indicates the SEM across subjects $(n=8)$.
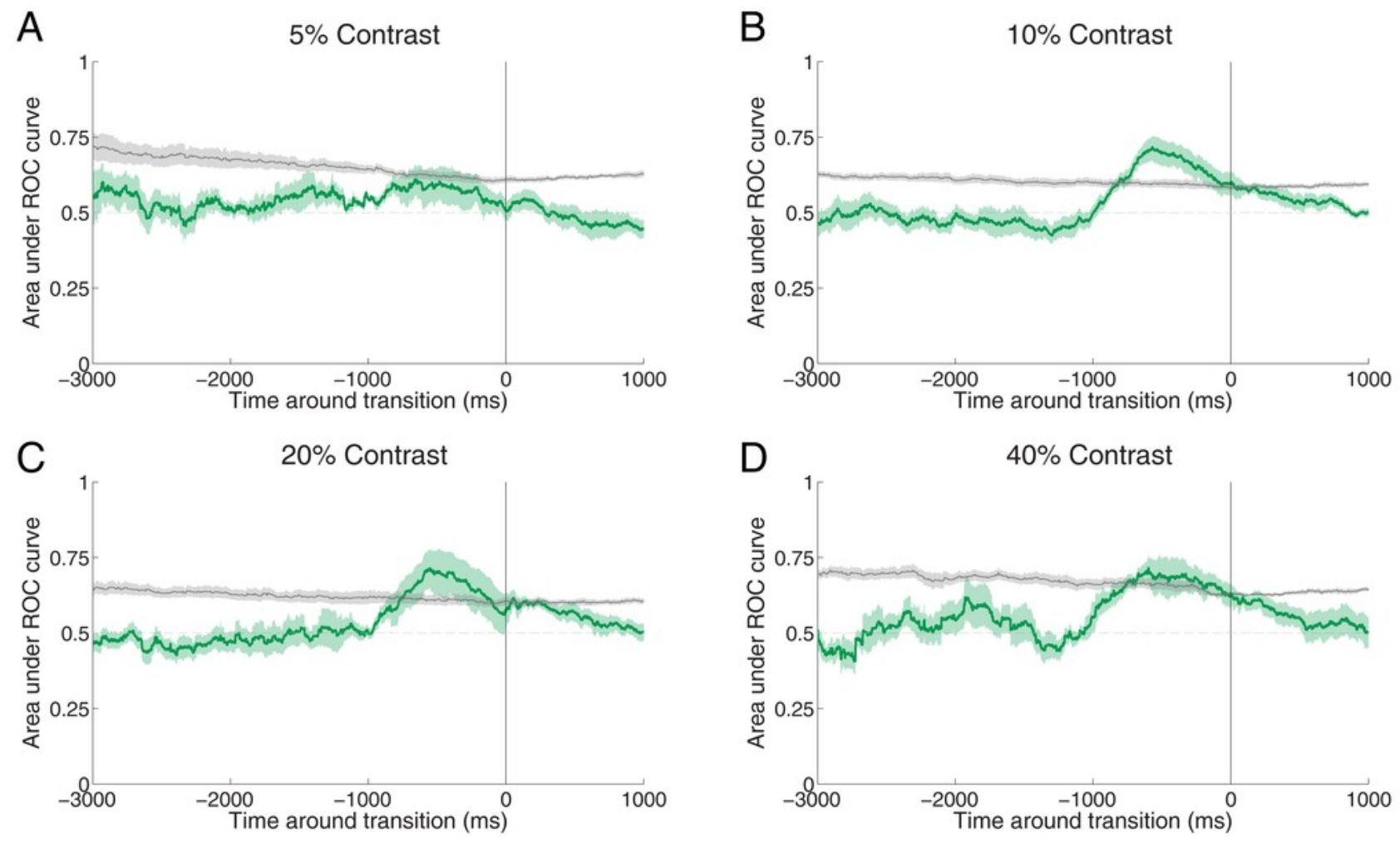